\title{
Shower reconstruction performance of the new Tibet hybrid experiment consisting of YAC-II, Tibet-III and MD arrays
}

\author{
D. Chen ${ }^{*, a}$, J. Huang ${ }^{b}$, Ying Zhang ${ }^{b}$, L. M. Zhai ${ }^{b, a}$, Xu Chen ${ }^{b}$, M. Shibata ${ }^{c}$, \\ Y. Katayose ${ }^{c}$, X. B. Hu ${ }^{b, d}$ and Y. H. Lin ${ }^{b}$ \\ ${ }^{a}$ National Astronomical Observatories, Chinese Academy of Sciences, Beijing 100012, China \\ ${ }^{b}$ Key Laboratory of Particle Astrophysics, Institute of High Energy Physics, Chinese Academy of \\ Sciences, Beijing 100049, China \\ ${ }^{c}$ Faculty of Engineering, Yokohama National University, Yokohama 240-8501, Japan \\ ${ }^{d}$ Department of Physics, Shandong University, Jinan 250100, China
}

E-mail: chendingdbao.ac.cn

\begin{abstract}
A new hybrid detector system has been constructed by the Tibet ASgamma collaboration in Tibet, China, since 2014 to measure the chemical composition of cosmic rays in the wide energy range including the knee. The new detector system consists of an AS-core detector-grid (YAC-II) to detect a bundle of high-energy shower particles, the Tibet-III AS array and a MD cluster (large underground water-Cherenkov Muon-Detector cluster). Its goals is to reconstruct the primary energy and composition of cosmic rays at the energies between $50 \mathrm{TeV}$ to $10^{16} \mathrm{eV}$ thereby allowing a detailed investigation of the expected proton knee, helium knee and iron knee. In this paper, we present the accuracy of the shower reconstruction methods based on full Monte Carlo simulations. Implications to the discrimination power of the obtained parameters with respect to the nature of the primary particles has been considered.
\end{abstract}

The 34th International Cosmic Ray Conference,

30 July- 6 August, 2015

The Hague, The Netherlands

${ }^{*}$ Speaker. 


\section{Introduction}

The all-particle energy spectrum of primary cosmic rays is well characterized by a power law $\mathrm{dN} / \mathrm{dE} \propto \mathrm{E}^{-\gamma}$ over many orders of magnitude, with $\gamma$ changes sharply from 2.7 to 3.1 at about 4 $\mathrm{PeV}$ [四]. Such structure of the all-particle energy spectrum is called the "knee", which is considered to be closely related to the origin, acceleration and propagation mechanism of cosmic rays. In order to explain the existence of the knee, many hypotheses and mechanisms have been proposed. Although all these approaches can well describe the knee structure, there are much discrepancies in the prediction of the individual components at the knee region [ [ $]$ ], [B]. The difficulty of settling discussions is due to a lack of the data on the energy spectra of individual CR components whose energies are too high to catch directly with detectors onboard balloons and satellites because of extremely scarce flux. To challenge the measurement of chemical composition around the knee, we have developed a new air-shower core detector (YAC). One important improvement is to lower the detection threshold energy of primary particles to $\sim 10 \mathrm{TeV}$, about one order of magnitude smaller than the previous experiment [四]. With this improvement, the energy spectra of individual components measured by YAC will overlap with those of direct measurements [ [వ], which may help us to examine the knee of light component, such as "proton knee" or "helium knee". Another important improvement of YAC is its ability to count the number of shower particles passing through each detector in a wide dynamic ranging from 1 to $10^{6}$ particles, making it possible to observe the primary cosmic rays in the energy range from $\sim 10 \mathrm{TeV}$ to $\sim 10 \mathrm{PeV}$. Its goals is to reconstruct the primary energy and composition of cosmic rays at the energies between $50 \mathrm{TeV}$ to $10^{16} \mathrm{eV}$ thereby allowing a detailed investigation of the expected "proton knee", "helium knee" and "iron knee". In this paper, we present the accuracy of the shower reconstruction methods based on full Monte Carlo simulations. Implications to the discrimination power of the obtained parameters with respect to the nature of the primary particles has been considered.

\section{2. (Tibet-III + YAC-II + MD) experiment}

The new Tibet hybrid experiment (Tibet-III+YAC-II+MD) has been operated in Tibet, China, and data taking started from February 2014. This hybrid experiment currently consists of three types of detector array, including the Tibet AS array (Tibet-III), the Yangbajing AS core-detector array (YAC-II) and an underground water-Cherenkov muon-detector array (MD), as shown in Fig. $\mathrm{W}$. The Tibet-III consists of 789 detectors, with a covering area $\sim 50,000 \mathrm{~m}^{2}$; the YAC-II consists of 124 detectors with a covering area of $\sim 500 \mathrm{~m}^{2}$ and the five MD pools consists of 80 cells, with an area of $\sim 4500 \mathrm{~m}^{2}$. The inner 100 plastic scintillator units of YAC-II are arranged as an array $(10 \times 10$ grid) each with an area of $50 \mathrm{~cm} \times 80 \mathrm{~cm}$, with $1.9 \mathrm{~m}$ spacing; and the outer 24 plastic scintillator units are arranged around the inner array each with an area of $50 \mathrm{~cm} \times 100 \mathrm{~cm}$. The outer 24 units are used to reject non core events whose shower cores are far from the YAC-II array. A lead plate of the thickness $3.5 \mathrm{~cm}$ is put on each YAC-II detector to convert high energy electrons and gammas in the cores into electromagnetic showers. Light signals from each scintillator are read out using two photomultiplier tubes of high gain (HAMAMATSU: R4125) and low gain (HAMAMATSU: R5325) to cover the wide dynamic range from 1 MIP (Minimum Ionization Particle) to $10^{6}$ MIPs. The YAC-II array has been constructed near the center of the Tibet-III, and operating 
simultaneously with it. For an air shower event, the Tibet-III provides the arrival direction $(\theta)$, and the air shower size $\left(N_{e}\right)$ which is interrelated to primary energy, the YAC-II measures the high energy electromagnetic particles in the core region so as to obtain the characteristic parameters of air-shower cores. When a YAC event is triggered, its accompanying air shower is simultaneously recorded. The matching between YAC, AS and MD event is made by their arrival time stamps. The air-shower direction can be estimated with an error smaller than $0.2^{0}$ above $100 \mathrm{TeV}$, and the primary energy resolution is estimated to be $12 \%$ at energies around $10^{15} \mathrm{eV}$ by our simulation.
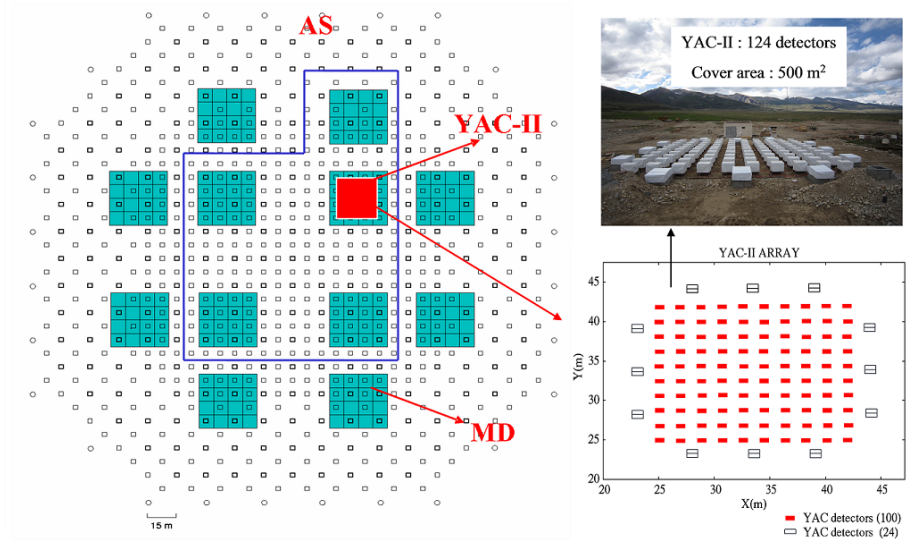

Figure 1: Schematic view of (Tibet-III+YAC-II+MD) array

\section{Monte Carlo Simulation}

A full Monte Carlo (MC) simulation has been carried out on the development of air showers in the atmosphere using the simulation code Corsika(version 6.204) [四] including the interaction models QGSJET01C and SIBYLL2.1. For the primary cosmic rays, we examined three primary composition models [ [ [ , 国], namely, "He-poor", "He-rich" and "Gaisser-fit" models, in order to evaluate the systematic errors attributable to primary composition models. Primaries isotropically incident at the top of the atmosphere within the zenith angles from 0 to 60 degrees are injected into the atmosphere. The detector responses to shower particles falling on the detectors of (YAC-II+Tibet-III+MD) array are calculated using the Geant4 [Q], where the detector performance, trigger efficiency and effective area are adequately taken into account based on the experimental conditions. The simulated data were analyzed in the same manner as in the procedure for the experimental data analysis.

\section{Analysis}

\subsection{Reconstruction of air-shower events}

The same procedure as the experimental data analysis for air showers was applied to the MC data, i.e., the reconstruction of the core position, the arrival direction are described in [四. In this work, the determination of the lateral distribution function of shower particles is very important, 
$1.0 \leq \sec (\theta)<1.1 \quad 1.0 \leq$ age $<1.1$

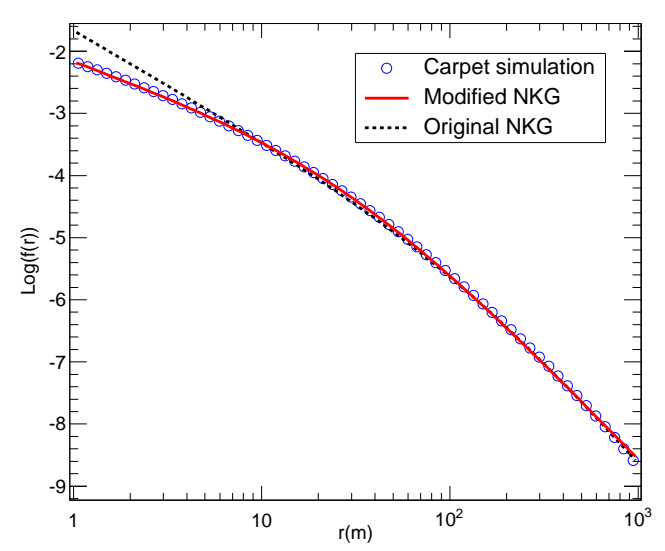

Figure 2: Lateral density distribution of the charge particles obtained with use of the carpet simulation. It is confirmed that the lateral distribution of the shower particles is better reproduced by our modified NKG function.

since the total number of charged particles in each event is estimated by fitting this function to the experimental data. Using the MC data obtained under the same conditions as the experiment, we found that the following modified NKG function can be well fitted to the lateral distribution of shower particles obtained with the detectors putting a lead plate of $5 \mathrm{~mm}$ thickness on its surface.

$$
\begin{aligned}
& f(r, s)=\frac{N_{e}}{C(s)}\left(\frac{r}{r_{m}{ }^{\prime}}\right)^{a(s)}\left(1+\frac{r}{r_{m}{ }^{\prime}}\right)^{b(s)} / r_{m}{ }^{2} \\
& C(s)=2 \pi B(a(s)+2,-b(s)-a(s)-2)
\end{aligned}
$$

where $r_{m}{ }^{\prime}=30 \mathrm{~m}$, and the variable $s$ corresponds to the age parameter, $N_{e}$ the total number of shower particles and $B$ denotes the beta function. The functions $a(s)$ and $b(s)$ are determined as follows. In CORSIKA simulation, the shower age parameter $s$ is calculated at observation level by fitting to a function for the one dimensional shower development. It may be possible to assume that air showers with the same shower age s are in the almost same stage of air shower development in the atmosphere, i.e. they show the almost same lateral distribution for shower particles irrespective of their primary energies. The lateral distribution of the particle density obtained by the simulation with carpet array configuration is normalized by the total number of particles which is derived from the total energy deposit in infinitely wide scintillator. These events are then classified according to the stage of air shower development using the age parameter and they are averaged over the classified events. The fitting of the equation (H. I) to the averaged MC data is made to obtain the numerical values $a$ and $b$ [四]. It is confirmed that the lateral distribution of the shower particles is better reproduced by our modified NKG function ( see Fig. $\square$ ).

Based on the Monte Carlo simulation, the correlation between the true shower size $\left(\mathrm{Ne}_{\text {true }}\right)$ and the estimated shower size $\left(N e_{f i t}\right)$ is demonstrated in Fig. B(left figure). Here, the true shower size $\left(N e_{\text {true }}\right)$ means particle number calculated for carpet array while estimated shower size $\left(N e_{f i t}\right)$ for real Tibet-III array using the above modified NKG function. As seen in Fig. B(left figure), a good correlation between the true shower size and the estimated shower size, and we found that the 
shower size resolutions estimated is about $5 \%$ around the primary energy of $1000 \mathrm{TeV}$ as seen in Fig. [1](right figure).
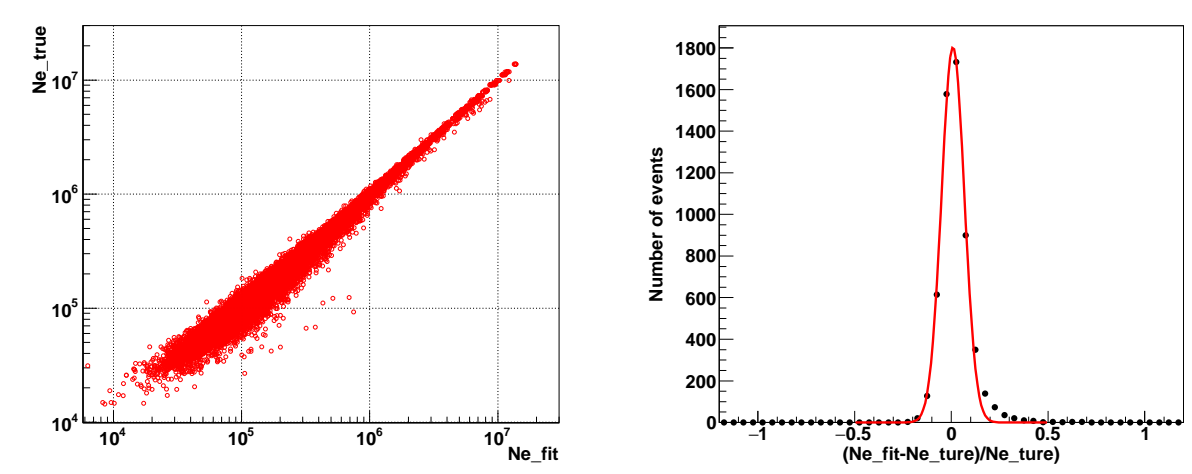

Figure 3: Left: Correlation between the true shower size $\left(N e_{\text {true }}\right)$ and the estimated shower size $\left(N e_{f i t}\right)$; Right: Shower size resolution is estimated to be $5 \%$ around the primary energy of $1000 \mathrm{TeV}$ based on the SIBYLL2.1 + He-poor model.

We are then able to obtain the correlation between the estimated shower size $N_{e}$ and the primary energy $E_{0}$ for each interaction models and composition models. The conversion function from the shower size $N_{e}$ to the primary energy $E_{0}$ can be expressed by the following equation for $\sec \theta$ $\leq 1.1$,

$$
E_{0}=\alpha \times N_{e}^{\beta},
$$

where $\alpha$ and $\beta$ are the parameters fitted through MC simulation. The values of $\alpha$ and $\beta$ are different for different primary cosmic-ray nuclei or mass groups. Fig.(1/ (left figure) shows the correlation between primary energy and shower size of the (proton+helium)-like events separated by ANN [ [D]. The solid line shows the fitting result of (proton+helium)-like events (based on SIBYLL2.1+He-poor model). It is expressed as $E_{0}=1.94 \times N_{e}{ }^{0.948} \mathrm{GeV}$ and the corresponding energy resolution calculated as about $12 \%$ at around $1000 \mathrm{TeV}$ as shown in Fig.(1) (right figure). We also checked the hadronic interaction model dependence in the correlation of $N_{e}$ and $E_{0}$, and obtained the similar relationship in QGSJET01c+He-poor model as $E_{0}=1.95 \times N_{e} e^{0.949} \mathrm{GeV}$. We found there is less than $3 \%$ difference for the determination of the primary energy based on different hadronic interaction models.

\subsection{Reconstruction of air-shower core events}

The reconstruction of air-shower core events will mainly start from the evaluation of the number of burst size $\left(N_{b}\right)$ under the lead plate of a YAC detector unit, that is the total energy loss in each scintillator divided by the single peak value, and we can obtain the following parameters reflecting the features of the core region using the YAC-II detector:

$N_{\text {hit }}$ - the number of "fired" detector units with $N_{b} \geq$ a given threshold value ( $N_{b} \geq 100$ in this paper);

$N_{b}{ }^{\text {top }}$ - the maximum burst size among the fired detectors;

$\Sigma N_{b}$ - the total burst size of all fired detector units; 

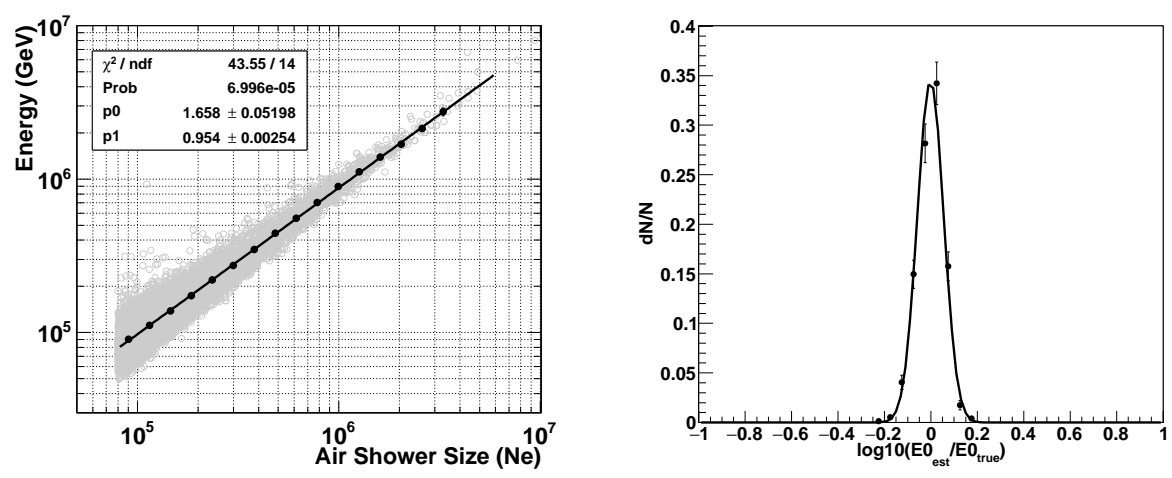

Figure 4: Left: Scatter plots of the primary energy (E0) and the estimated size (Ne) in case of the SIBLL2.1 + He-poor model; Right: Energy resolution is estimated to be $12 \%$ around the primary energy of $1 \mathrm{PeV}$ in case of the SIBLL2.1 + He-poor model.

$\langle R\rangle$ - the mean lateral spread, $\langle R\rangle=\sum r_{i} /\left(N_{h i t}-1\right)$;

$\left\langle N_{b} R\right\rangle$ - the mean energy-flow spread, $\left\langle N_{b} R\right\rangle=\sum\left(N_{b i} \times r_{i}\right) / N_{h i t}$, where $N_{b i}$ and $r_{i}$ are the burst size in the $i^{\text {th }}$ fired detector unit and the lateral distance from the burst center, which is calculated as $N_{b}$ weighted center of gravity, to the center of the $i^{t h}$ fired detector, respectively.

We also tried to reconstruct other parameters reflecting the features of core region, and found that the five parameters mentioned above are basic and enough for the separation of primary cosmic-ray mass, other parameters bring slight improvement to the separation of primary cosmicray mass.

\subsection{Reconstruction of Muon events}

Each cell of the MD array is composed of a concrete water tank of $7.2 \mathrm{~m}$ wide $\times 7.2 \mathrm{~m}$ long $\times 1.5 \mathrm{~m}$ deep, equipped with two downward-facing 20-inch-in-diameter PMTs (HAMAMATSU: R3600) on the ceiling. The reconstruction of the muon events was made by calculating the number of Cherenkov photons hitting on the PMTs, then we can get the number of muons at each MD pool as the total number of photons divided by the single peak value. Finally, we get the sum of muons $\left(N_{\mu}\right)$ "fired" in the MD array of an air-shower event.

\section{Results and Discussions}

Based on the above section 4.1, 4.2 and 4.3, we can obtain some parameters recorded by this new Tibet hybrid experiment (YAC-II+Tibet-III+MD) and it is found they are very sensitive to different primary cosmic-ray nuclei. An example of the sensitivity to the primary mass by the parameters from YAC-II array is shown in Fig.5(left figure), in which a correlation between $N_{\text {hit }}$ and $N_{b}{ }^{t o p}$, both of them are normalized by $N_{e}$, is shown by different color for Proton+Helium, Middle and Iron nuclei under QGSJET01c+He-poor model. For the MD array, the sensitivity to the primary nuclei is seen in $N_{e}$ and $N_{\mu}$ correlation. Fig. \ (middle figure) shows the correlation between $N_{e}$ and $N_{\mu}$ on each event observed with the (YAC-II+Tibet-III+MD) array based on QGSJET01c+He-poor model, and we can see that the distribution of $N_{\mu}$ and $N_{e}$ apparently depends on the primary nuclei. 

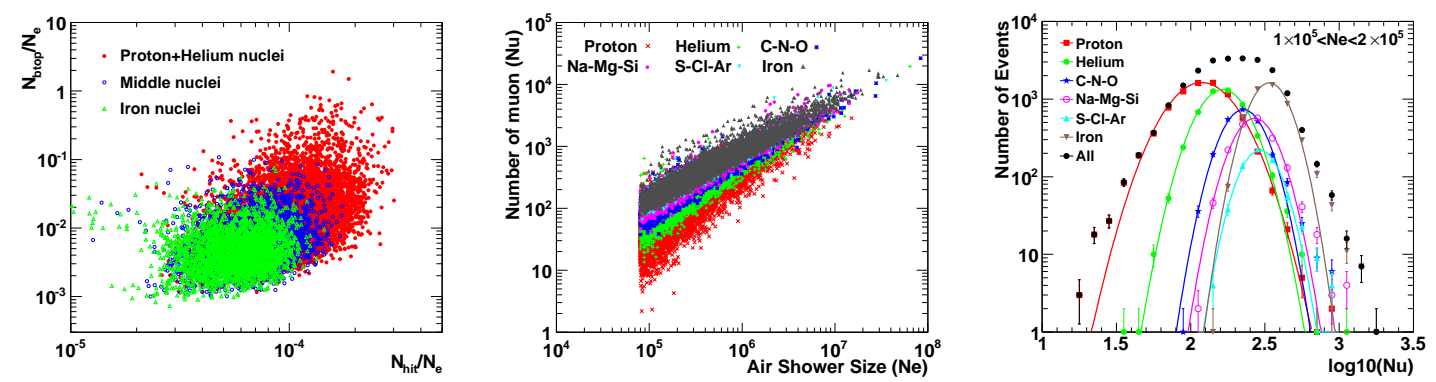

Figure 5: Left: The correlation between the $\mathrm{N}_{h i t}$ and $N_{b}{ }^{\text {top }}$ parameters under QGSJET+He-poor model, both of which are normalized by Ne; Middle: The correlation between $\mathrm{Ne}$ and $N_{\mu}$ observed with the ( YAC-II+Tibet-III + MD ) array; Right: $N_{\mu}$ distribution for various primary nuclei at $1 \times 10^{5}<N_{e}<2 \times 10^{5}$ bin.

When we set $1 \times 10^{5}<N_{e}<2 \times 10^{5}$, the $N_{\mu}$ distribution strongly depends on primary mass as shown in the Fig.5(right figure).

We also can obtain some parameters reflecting the features of AS core region under different hadronic interaction models and primary cosmic-ray composition models as shown in Fig.6, and we found that the shapes of the parameters are almost the same. We also checked the distribution of other parameters, such as $\sum N_{b},\left\langle N_{b} R\right\rangle, N_{e}$, etc., and obtained the same results. Such situation indicates that the characteristics of the AS core event are well observed by our new hybrid array with weak dependences on the hadronic interaction models and primary composition models.

In this work, we use a feed-forward artificial neural network ( ANN) method to separate Plike events, He-like events and Iron-like events by using the above reconstructed parameters. MC shows that this new hybrid experiment has a capability of measuring the primary energy spectrum for each of 4 mass groups of $\mathrm{P}, \mathrm{He}$, Iron and $4<\mathrm{A}<56$ at $50 \mathrm{TeV}-10 \mathrm{PeV}$ range covering the knee.

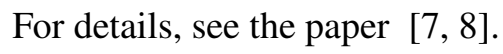

\section{Summary}

In this paper, we present the accuracy of the shower reconstruction methods based on full Monte Carlo simulations. Implications to the discrimination power of the obtained parameters with respect to the nature of the primary particles has been considered. We also examined the possibility and sensitivity to measure the primary CR composition and the spectrum for each component in the energy range from $50 \mathrm{TeV}$ to $10 \mathrm{PeV}$ with the new Tibet hybrid detector system operating at Yangbajing in Tibet since 2014 based on the full MC simulation. It is shown that YAC well works together with other detectors to achieve our purpose and is able to observe the energy spectrum of each of 4 mass groups of $\mathrm{P}, \mathrm{He}$, Iron and $4<\mathrm{A}<56$ separately in the above energy region. The energy resolution of primary CRs better than $12 \%$ at $10^{15} \mathrm{eV}$ may guarantee to detect the break energies of the power spectra of protons and helium nuclei in the knee region. This is indispensable to deepen our understanding on the origin and acceleration mechanism of primary CRs up to the knee energy.

\section{Acknowledgments}

The authors would like to express their thanks to the members of the Tibet AS $\gamma$ collabora- 

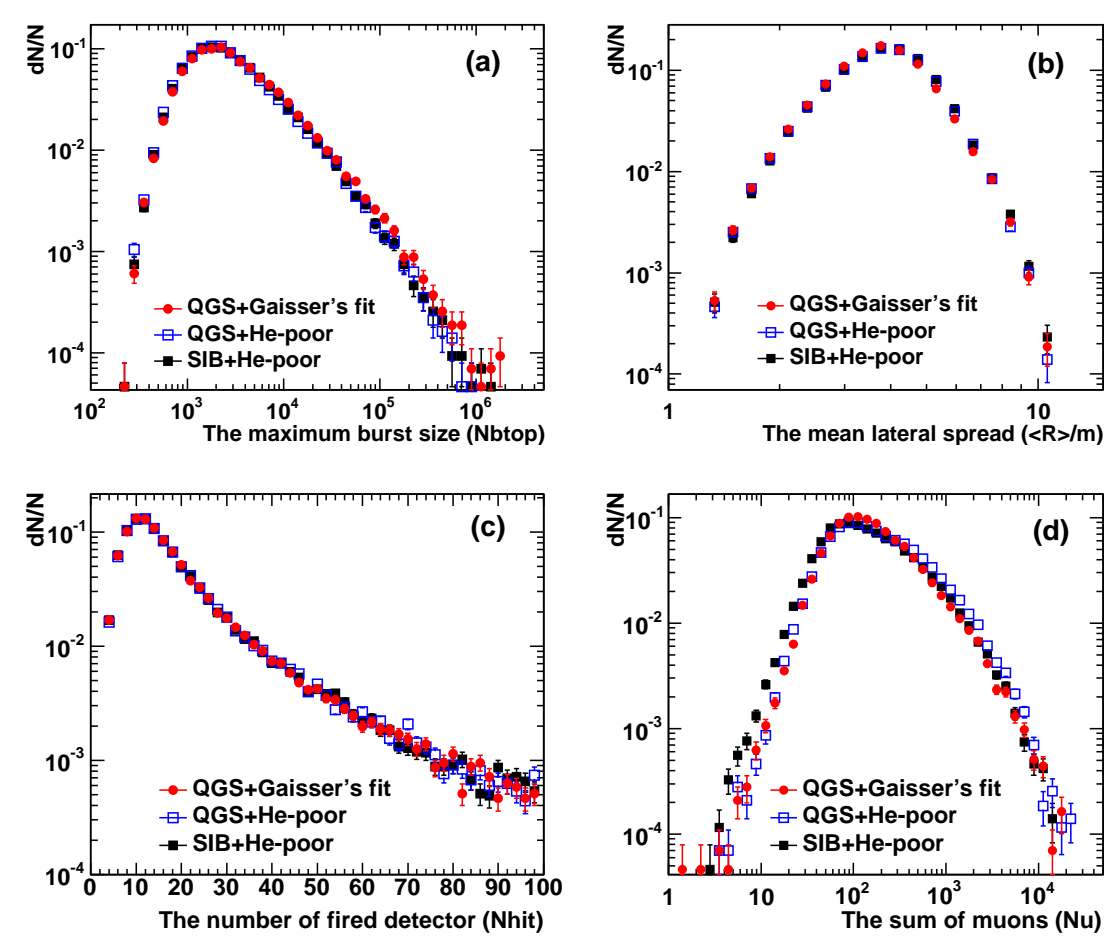

Figure 6: The distribution of some parameters((a): $N_{b}{ }^{t o p}$, (b): $\langle R\rangle,(\mathrm{c}): N_{\text {hit }}$ are given by YAC-II array, (d): $N_{\mu}$ is given by the MD array) of high-energy core events under different hadronic interaction models and primary cosmic-ray composition models [四].

tion for the fruitful discussion. This work is supported by the Grants from the National Natural Science Foundation of China (11078002 and 11275212) and the Chinese Academy of Sciences (H9291450S3, 2013T2J0006) and the Key Laboratory of Particle Astrophysics, Institute of High Energy Physics, CAS. The Knowledge Innovation Fund (H95451D0U2 and H8515530U1) of IHEP, China also provide support to this study.

\section{References}

[1] M. Amenomori et al., ApJ 678 (2008) 1165.

[2] J. R. Hörandel, Astropart. Phys. 19 (2003) 193.

[3] M. Shibata, Y. Katayose, J. Huang and D. Chen, ApJ 716 (2010) 1076.

[4] M. Amenomori et al., Phys. Lett. B 632 (2006) 58.

[5] Y. S. Yoon, et al., ApJ 728 (2011) 122.

[6] Heck D. et al., 1998 Forschungszentrum Karlsruhe Report FZKA 6019

[7] J. Huang et al., Astropart. Phys. 66 (2015) 18.

[8] L. M. Zhai et al., J. Phys. G: Nucl. Part. Phys. 42 (2015) 045201.

[9] Agostinelli S. et al., Nucl. Instrum. Meth. Phys. Res. A 506 (2003) 250. 